\title{
First-principle diffraction simulations as a tool to solve the nanodiffraction problem
}

\author{
H. Öztürk' ${ }^{1}$ I.C. Noyan² \\ ${ }^{1}$ Özyeğin University, Department of Mechanical Engineering, Çekmeköy, İstanbul, TR, \\ ${ }^{2}$ Columbia University, Department of Applied Physics and Applied Mathematics, New York, USA \\ hande.ozturk@ozyegin.edu.tr
}

\begin{abstract}
Computer simulations are being increasingly used to understand the diffraction phenomenon from nanomaterials. Typically, such simulations are performed with the goal of establishing a mathematical relationship between the diffracting material and its diffraction profile under certain assumptions. For simulation of powder diffraction, the famous Debye equation [1] is generally used which also relies on particular assumptions about the diffracting material such as all Bragg reflections being represented by enough number of particles in the ensemble [2]. In this talk we will describe an alternative methodology that relies only on the far-field diffraction formulation [3] and starts off from the scattering phenomenon of $\mathrm{x}$-rays from individual atomic positions. This methodology will be shown to be powerful and more general than the Debye equation -by relaxing some of the implicit requirements imposed by the Debye formula- enabling direct connection between each diffracted spot on a 2D detector and the diffracting crystallites $[4,5]$. Once the methodology is explained, example studies on nanodiffraction experiments will be introduced and new information obtained by the computational tool will be demonstrated [6]. Although the proposed computational methodology is quite time-consuming since large number of calculations need to be performed for simulating diffraction from relatively larger nanocrystals, parallellization algorithms combined with exponentially increasing computational power becoming much more available to most researchers will potentially popularize its use in nanocharacterization studies in the near future.
\end{abstract}

[1] Debye, P. (1915). Annalen der Physik. 46, 809-823.

[2] Bertolotti, F., Moscheni, D., Migliori, A. et al. (2016). Acta Crystallographica Section A.,72, 632-644.

[3] Warren, B. E. (1990). X-ray Diffraction. Dover Books on Physics. Courier Dover Publications.

[4] Öztürk, H, Yan, H., Hill J. \& Noyan, I. C. (2014). Journal of Applied Crystallography, 47, 1016-1025.

[5] Öztürk, H, Yan, H., Hill J. \& Noyan, I. C. (2015). Journal of Applied Crystallography, 48, 1212-1227.

[6] Xiong, S., Öztürk, H, Lee, S-Y., Mooney P., \& Noyan, I. C., Journal of Applied Crystallography, 51, 1102-1115.

\section{Keywords: Powder diffraction; nanocrystals; x-rays; computer simulations}

Any acknowledgements authors wish to make should be included at the end of the manuscript with no heading (use Acknowledgement style, Times New Roman 10 pt, italics). 